\title{
Evaluating experimental methods to manipulate authentic vs. hubristic pride: Four pilot studies
}

\author{
Kodai Kusano $^{1}$, Markus Kemmelmeier ${ }^{1}$ \\ ${ }^{1}$ Interdisciplinary Social Psychology Ph.D. Program, \\ University of Nevada, Reno, USA
}

\begin{abstract}
Summary Inducing different types of pride is an experimental challenge. Four studies varied whether participants felt authentic or hubristic pride, two using an autobiographical recall task and two using a false-feedback paradigm. Ratings of authentic pride did not vary as a function of the type of emotion induction, though ratings of hubristic pride items did. Other evidence demonstrated that socially desirable responding depressed self-ratings of hubristic pride. Keywords · pride $\cdot$ attribution $\cdot$ sex difference $\cdot$ emotion induction ·manipulation Kodai Kusano: kk@nevada.unr.edu
\end{abstract}

Introduction Authentic pride arises when personal success originates from malleable characteristics (e.g. effort), whereas bubristic pride arises when success originates from static traits (e.g., heritage) (Tracy \& Robins, 2007). When researchers try to experimentally induce different types of pride, there are three challenges: (1) people report lower hubristic pride than authentic pride regardless of manipulation; (2) socially desirable responding lowers reported feelings of hubristic pride; and (3) though attribution to malleable or static characteristics is at the heart of the authentic-hubristic pride distinction, theory-focused experimental manipulations that vary the cause of success seem to be untested.

\begin{tabular}{|c|c|c|c|c|c|}
\hline & & \multicolumn{4}{|c|}{ Experimental Conditions } \\
\hline & & \multicolumn{2}{|c|}{ Authentic } & \multicolumn{2}{|c|}{ Hubristic } \\
\hline & Ratings: & Authentic & Hubristic & Authentic & Hubristic \\
\hline Study 1 & (Self) & 5.33 & 2.13 & 5.46 & 2.15 \\
\hline Study 2 & (Self) & 4.34 & 1.33 & 4.19 & 1.95 \\
\hline Study 3 & (Self) & 4.93 & 1.55 & 4.71 & 1.86 \\
\hline \multirow[t]{2}{*}{ Study 4} & (Self) & 5.4 & 1.75 & 5.52 & 1.93 \\
\hline & (Other) & 5.63 & 2.92 & 5.76 & 3.05 \\
\hline
\end{tabular}

Table 1: Mean ratings of authentic vs. hubristic pride across four studies by authentic vs. hubristic condition.

Aims Our goal was to discern a reliable method to induce authentic vs. hubristic pride by contrasting procedures used to induce both types of pride. We compared two versions of an autobiographical recall task: without explicit encouragement (Study 1) and with encouragement to report even socially undesirable feelings (Study 2; Ashton-James \& Tracy, 2012). We also developed a false-feedback paradigm to have participants feel pride about their abilities concerning interpersonal perception (Study 3; Williams \& DeSteno, 2009) and their leadership aptitude (Study 4; Fast \& Chen, 2009). Because attribution is at the heart of the distinction between hubristic and authentic pride, Study 3 and 4 kept the task constant, but varied whether the cause of participants' success was malleable or static. In Study 4, we also asked participants to predict the responses of others to the emotion-induction tasks.

Method Study $1(n=200)$ asked participants to recall a situation in which they had either experienced authentic pride or hubristic pride. Study $2(n=35)$ used the same recall task but encouraged participants to express their feelings even if they are not socially desirable. Study $3(n=70)$ provided participants with (false) positive feedback in response to an engaging interpersonal perception task, with participants attributing their success (a high score compared to other participants) to either malleable or static personal characteristics, with Study $4(n=196)$ presenting the same task as being diagnostic of leadership aptitude. In all studies, participants rated feelings of authentic pride and hubristic pride, though in Study 4 they also predicted how others would feel.

Results Across all four studies we compared self-ratings of authentic pride and hubristic pride. Regardless of condition, participants' ratings of felt authentic pride exceeded selfratings of hubristic pride. When both types of ratings were submitted to a mini-meta-analysis, there was no difference in self-reported authentic pride as a function of which type of pride was induced (Mean $d=-.04$, range $=-.12$ to .24 ), though self-reported hubristic pride was higher in the hubristic pride conditions (Mean $d=.16)$. The largest effect size $(d=.71)$ emerged for Study 2, where participants had been encouraged to express socially undesirable emotion, and the next largest effect size was in Study $3(d=.31)$. However, in Study 4 participants predicted that others would not necessarily experience higher levels of hubristic pride in the hubristic pride condition vs. authentic pride condition $(d=.08)$; yet, they predicted that others would experience much more hubristic pride in the hubristic pride condition than they were willing to admit themselves (difference in other-self ratings, $d=.81$ ). Across the four studies, men reported higher levels of hubristic pride (Mean $d=.27$ ), and their reported hubristic pride was more sensitive to the experimental manipulation $(d=.21)$, than that of women $(d=.15)$.

Conclusions The autobiographical recall task with explicit encouragement to report a pride-inducing episode (Study 2) was most effective in differentiating authentic pride from hubristic pride. Yet, ratings of hubristic pride were generally low across studies (mean $=2.16$ in 7-point Likert scale), and authentic pride systematically covaried with ratings of hubristic pride. The difficulty of inducing hubristic pride was partially due to socially-desirable responding, but even when participants predicted reactions of others, ratings of hubristic pride tended to be still low (mean $=2.99$ ). We suspect that there is substantial individual variability in responding pride-inducing events, especially when experiencing and reporting feelings of hubristic pride. We partially clarified this possibility by revealing gender differences. Overall, our work generated more problems than solutions: more work is necessary to develop a stronger manipulation of hubristic pride without confounding it with authentic pride. Future research must also consider gender differences as well as other potential individual differences.

\section{References}

Ashton-James, C. E., \& Tracy, J. L. (2012). Pride and prejudice: How feelings about the self influence judgments of others. Personality and Social Psychology Bulletin, 38(4), 466-476.

Tracy, J. L., \& Robins, R. W. (2007). The psychological structure of pride: A tale of two facets. Journal of Personality and Social Psychology, 92(3), 506525.

Williams, L. A., \& DeSteno, D. (2008). Pride and perseverance: The motivational role of pride. Journal of Personality and Social Psychology, 94(6), 1007-1017.

Fast, N. J., \& Chen, S. (2009). When the boss feels inadequate: Power, incompetence, and aggression. Psychological Science, 20(11), 1406-1413. 